\title{
THE RAYLEIGH QUOTIENT AND DYNAMIC PROGRAMMING
}

\author{
RICHARD BELLMAN \\ Department of Mathematics, Electrical Engineering, and Medicine \\ University of Southern California \\ Los Angeles, California 90007 \\ U.S.A.
}

(Received August 8, 1978)

ABSTRACT. The puppose of this paper is to derive a nonlinear partial differential equation for which $\lambda$ given by (1.3), is one value of the solution. In Section 2 , we derive this equation using a straightforward dynamic programming approsch. In Section 3, we discuss some computational aspects of derermining the solution of this equation. In Section 4, we show that the same method may be applied to the nonlinear characteristic value problem. In Section 5, we discuss how the method may by applied to find the higher characteristic values. In Section 5, we discuss how the same method may be applied to some matrix problems. Finally, in Section 7 , we discuss selective computation.

KEY WORDS AND PHRASES. Rayleigh Quotient, and Dynamic Programming. AMS (MOS) SUBJECT CLASSIFICATION (1970) CODES. 34.

1. INTRODUCTION.

Consider the equation 


$$
\mathbf{u}^{\prime \prime}+\lambda \mathbf{a}(t) \mathbf{u}=0
$$

where wave the boundary conditions

$$
u(0)=u(T)=0
$$

We shall assume that $a(t)$ is positive throughout the interval and possesses a Taylor expansion in the neighborhood of 0 . We know that the characteristic values are real, positive, and simple.

The smallest characteristic value is given by the Rayleigh quotient

$$
\lambda=\min \frac{\int_{0}^{T} u^{\prime 2} d t}{\int_{0}^{T} a(t) u^{2} d t},
$$

where $u$ is subject to the same boundary conditions as above.

\section{DYNAMIC PROGRAMMING APPROACH.}

Let us consider the more general problem

$$
f(c, T)=\min _{u} \int_{0}^{T} u^{\prime 2} d t
$$

where $u$ is subject to

$$
\begin{aligned}
& u(T)=c, \quad u(0)=0, \\
& \int_{0}^{T} a(t) u^{2} d t=1 .
\end{aligned}
$$

We now make the decomposition

$$
\int_{0}^{\mathrm{T}}=\int_{\mathrm{T}-\Delta}^{\mathrm{T}}+\int_{\theta}^{\mathrm{T}-\Delta}
$$

In the second integral we make the renormalization

$$
u=\left(1-c^{2} \Delta / 2\right) v
$$


We thus obtain, to terms in $\Delta^{2}$,

$$
f(c, T)=v^{2} \Delta+\left(1-c^{2} \Delta\right) f(c+v \Delta, T-\Delta)
$$

Passing to the limit we obtain the nonlinear partial differential equation

$$
f_{T}=-c f-f_{c}^{2} / 4
$$

\section{COMPUTATIONAL ASPECTS.}

The nonlinear partial differential equation above cannot be solved routinely on a digital conputer. In the first place, we see that the solution becomes unbounded as $T \rightarrow 0$; In the second place, we see that there is a singularity as both $c$ and $\mathrm{T} \rightarrow 0$.

To obtain the numerical solution, we can use analysis to derive the solution for small $T$. This is consistant with the general principle that effective conputing needs accommodation of analysis and the arithmetic capabilities of the digital computer. We also use anaylsis to derive the solution for small $c$ and $T$. If we keep the constant term in the Taylor expansion of $a(t)$, we can solve the associated differential equation in terms of trigonometric functions; If we keep the first two terms we can solve the equation in terms of Bessel functions of order 1/3; If we keep the first three terms, we can solve the differential equation in term of cylinder functions. Since we have to use power series expansions at some stage, it is probably best to avoid special functions and carry through the whole calculation using power series.

Another approach to this problem is given in [1],

\section{NONLINEAR CHARACTERISTIC VALUE PROBLEMS.}

Let us consider the function of three variables defined by

$$
f\left(c_{1}, c_{2}, T\right)=\min _{u} \int_{0}^{T} u^{\prime}{ }^{2} d t
$$


where $u$ is subject to

$$
\begin{aligned}
& u(T)=c_{1} u(0)=0 \\
& \int_{0}^{T} g(u) d t=c_{2} .
\end{aligned}
$$

If $g$ is a power of $u$, we can use renormalization as above to eliminate the variable $c_{2}$. If we have

$$
g(u)=u^{2}+a u^{n}
$$

we can use renormalization and take $a$ as a state variable.

The same method may be applied to general isoperimetric problems, [2].

\section{HIGHER CHARACTERISTIC VALUES.}

Let us now turn to the determination of higher characteristic values. It will be sufficient to consider the second characteristic value. We know that there is orthogonality. Hence, we add the condition

$$
\int_{0}^{T} u_{1} d t=c_{2} .
$$

Here, $u_{1}$ is the first characteristic function. The dynamic programming approach given above determines the function too.

We can now proceed as above to obtain a nonlinear partial differential equation.

\section{MATRIX THEORY.}

If we have a symmetric matrix, we can obtain Rayleigh quotients for the smallest and largest characteristic values.

If the matrix has a particular structure, we can use a dynamic programming approach. Examples are found in [3].

Matrix problems are obatined if the Ritz-Galerkin method is used. We can use the methods of [4] to obtain upper and lower bounds. 
In many cases, the matrix is non-negative as well as symmetric. We can obtain upper and lower bounds from this fact. An example is given in [5].

\section{SELECTIVE COMPUTATION.}

In many cases, we only want a few values of the nonlinear partial differential equation. In that case, we can solve the associated variational problem instead.

\section{REFERENCES}

1. Bellman, R. On the Determination of Characteristic Values for a Class of Sturm-Liouville Problems Illinois J. Math. $\underline{2}$ (1958) 577-585.

2. Bellman, R. Introduction to the Mathematical Theory of Control Processes Vo1. II Academic Press, Inc., New York, 1971.

3. Bellman, R. Introduction to Matrix Analysis McGraw-Hill Book Company, New York, 1960; 2nd edition, 1970.

4. Bellman, R. Selective Computation - V: The Largest Characteristic Root of a Matrix Journal of Nonlinear Analysis, to appear. 5. Bellman, R. and R. Latter On the Integral Equation $\lambda f(x)=\int_{0}^{a} K(x-y) f(y) d y$.
Proc. Amer. Math. Soc. $\underline{3}$ (1952) 884-891. 


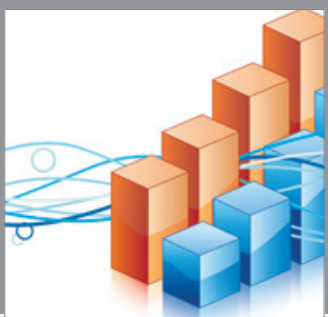

Advances in

Operations Research

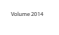

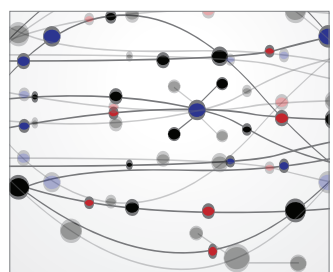

\section{The Scientific} World Journal
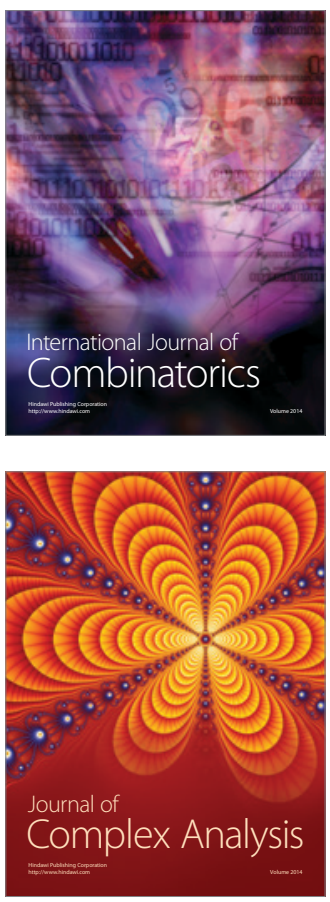

International Journal of

Mathematics and

Mathematical

Sciences
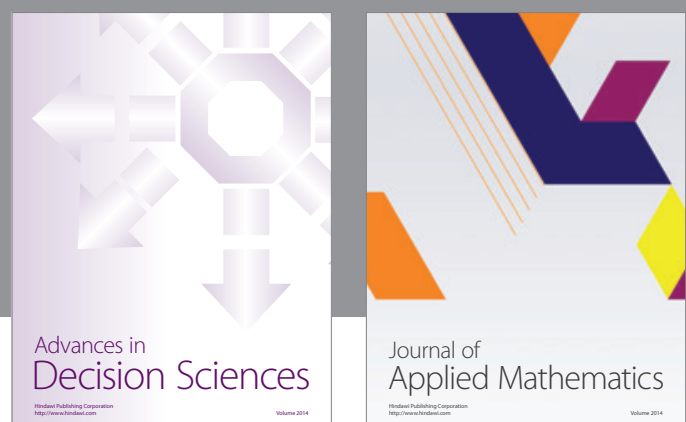

Journal of

Applied Mathematics
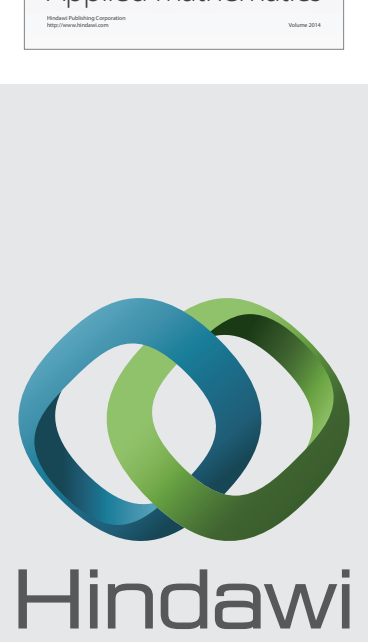

Submit your manuscripts at http://www.hindawi.com
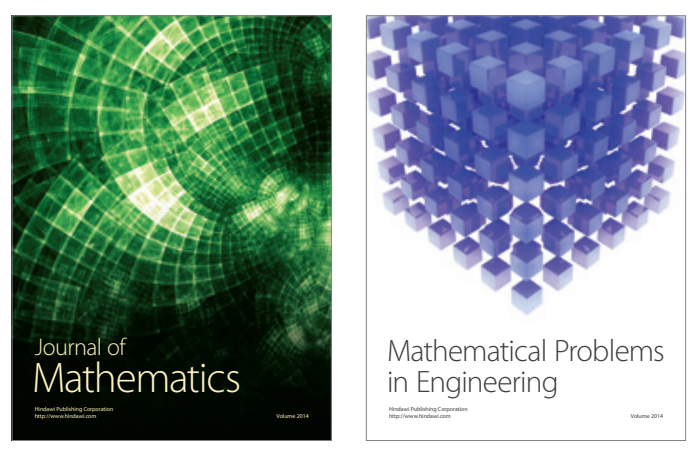

Mathematical Problems in Engineering
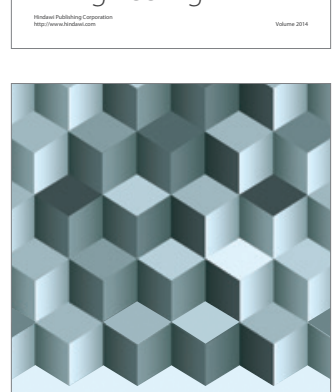

Journal of

Function Spaces
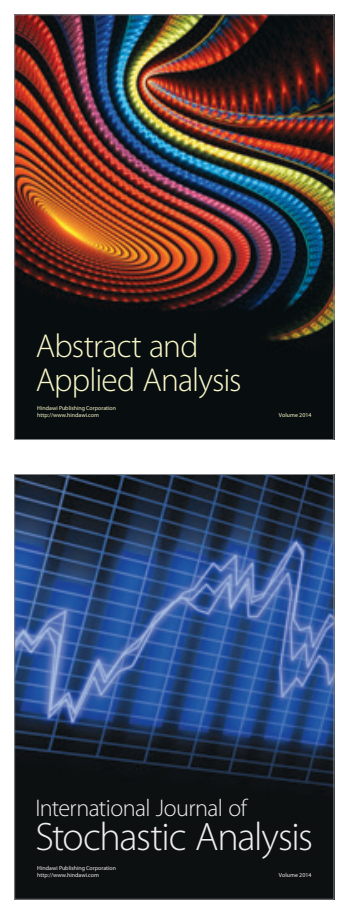

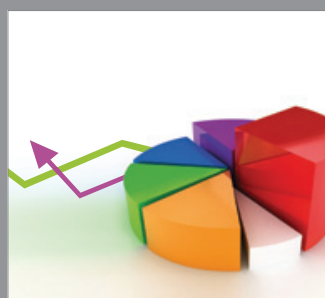

ournal of

Probability and Statistics

Promensencen
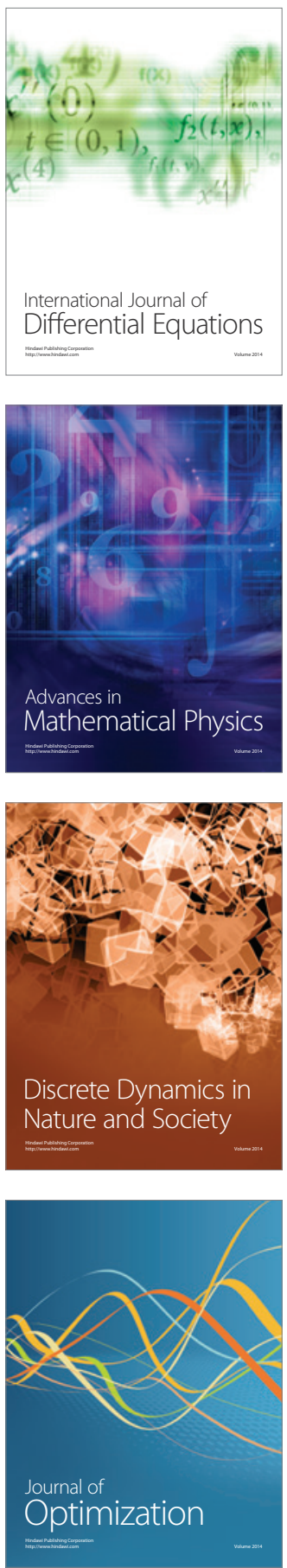